\title{
Diagnostic importance of bone marrow aspiration evaluation: A single-center study
}

\author{
Ali Dogan ${ }^{1}$, Sinan Demircioglu ${ }^{2}$
}

\begin{abstract}
Background \&Objective: Early diagnosis can be made based on the morphological examination of bone marrow aspiration (BMA) until the bone marrow biopsy (BMB) result is reported. This allows for treatment to be started immediately, especially in hematological malignancies for which urgent treatment is indicated. This study aimed to determine the sensitivity and importance of bone marrow aspiration in the diagnosis of hematological malignancies.

Methods: In this study, the data of patients who underwent bone marrow aspiration and bone marrow biopsy in Van Yuzuncu Yil University hospital between 2017 and 2019 were retrospectively analyzed. A total of 500 patients who simultaneously underwent BMA and BMB were included in the study. Data were obtained from electronic medical records.

Results: Indication for bone marrow evaluation was abnormalities in complete blood count in 270 (54\%) of patients. The diagnosis was made based on the evaluation of BMA in 475 (95\%). In 456 (96\%) of the 475 patients diagnosed with BMA, the diagnosis was consistent with that of $B M B$. Agreement of BMB with BMA was $100 \%$ in acute and chronic leukemias, while BMA was not sufficient for the diagnosis of lymphoma and solid organ metastasis.

Conclusion: Our study showed that the evaluation of BMA was highly sensitive in the diagnosis of hematological malignancies, such as acute leukemias, chronic leukemias, and multiple myeloma.
\end{abstract}

KEYWORDS: Bone marrow aspiration, Bone marrow biopsy, Evaluation.

doi: https://doi.org/10.12669/pjms.38.4.4797

How to cite this:

Dogan A, Demircioglu S. Diagnostic importance of bone marrow aspiration evaluation: A single-center study. Pak J Med Sci. 2022;38(4):811-815. doi: https://doi.org/10.12669/pjms.38.4.4797

This is an Open Access article distributed under the terms of the Creative Commons Attribution License (http://creativecommons.org/licenses/by/3.0), which permits unrestricted use, distribution, and reproduction in any medium, provided the original work is properly cited.

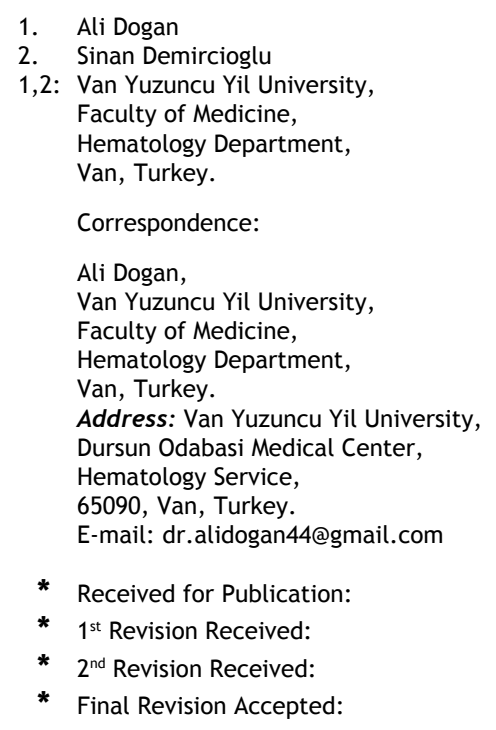

Pak J Med Sci
June 1, 2021

July 14, 2021

November 26, 2021

December 8, 2021

\section{INTRODUCTION}

As a large organ mass, bone marrow is not only the source of many hematological diseases but also a site of involvement of some infections, solid organ metastases, and metabolic diseases. ${ }^{1}$ Effects on bone marrow manifest in abnormal laboratory values, lymphadenopathy, splenomegaly, and disease-specific clinical symptoms. ${ }^{1}$ The examination of bone marrow in many blood diseases is an important diagnostic tool following the assessment of medical history, physical examination, blood count analysis, and examination of peripheral smear morphology. In such cases, it is of great importance to examine using bone marrow aspiration and bone marrow biopsy procedures, which are usually, performed simultaneously. ${ }^{2}$ 
BMA is mainly performed for the cytomorphological examination of bone marrow cells. It also allows taking blood samples for other analysis such as flow cytometry, cytogenetics, molecular genetics, microbiological tests, and immunophenotyping. ${ }^{3} \quad$ The morphological examination of BMA offers an accurate and early diagnosis of some hematological malignancies within a few hours until the result of $B M B$ is reported within an average of 10-14 days. Thus, treatment of life-threatening malignancies that require urgent treatment, such as acute and chronic leukemias and multiple myeloma can be started immediately. In this study, we aimed to investigate the sensitivity of BMA for the diagnosis of hematological malignancies and evaluate the compatibility between BMA and BMB.

\section{METHODS}

A total of 500 patients who underwent simultaneously BMA and BMB in Van Yuzuncu Yil University Medical Faculty Hospital between 2017 and 2019 were included in the study. Patients with and age $\geq 18$ were included. The patient files and data recorded in the hospital's information system were analyzed retrospectively. The bone marrow examination was performed from the posterior superior of the iliac crest and anterior superior of the iliac crest in patients. For BMA examination, $0.5 \mathrm{ml}$ of bone marrow blood was aspirated at the first aspirate. In addition, bone marrow biopsy material of $\geq 1$ centimeter was taken for $\mathrm{BMB}$ examination.

The demographic data, laboratory values of the patients, peripheral smear findings, bone marrow aspiration, and biopsy indications were examined. As a result of these preliminary evaluations, in patients with suspected hematological malignancies, blood samples for flow cytometry and genetic mutation analysis were taken from the area where BMA/BMB had been performed. BMB material sampled from the place where BMA had been performed or from a different area was transferred to the pathology laboratory in formol. The blood aspirated from bone marrow was spread on slides and stained with May-Grunwald-Giemsa staining, and then examined under a microscope at 10 and 100 magnifications. Cellularity and megakaryocytes were evaluated at 10 magnifications in the BMA examination. The morphology, maturation and differentiation characteristics of cells at 100 magnifications were determined by counting 400 cells and formulation. Subtyping of acute leukemias was made by evaluating flow cytometry. In cases where the examination of BMA was not sufficient, the definitive diagnosis was made based on the $\mathrm{BMB}$ result. The agreement between the diagnoses of BMA and BMB was assessed.

Statistical Analysis: Numerical data were expressed as mean \pm standard deviation, and categorical variables as numbers and percentages $(n, \%)$. In the evaluation of BMA and BMB, if the diagnosis was the same, the result was accepted as consisted, and if the diagnoses of the two procedures differed, the result was considered as inconsistent. The rate of agreement between the two procedures was expressed as a percentage. Statistical analyses were performed using SPSS version 25.0 (IBM SPSS, Chicago, IL).

Ethical approval: Ethical approval for the study was obtained from the non-invasive clinical research ethics committee of Van Yuzuncu Yil University (approval date: 05.07.2019, decision number: 2019/11-03).

Table-I: Bone marrow examination indications of the patients.

\begin{tabular}{lc}
\hline Indication & $n(\%)$ \\
\hline Laboratory findings & \\
Pancytopenia & $83(16.6 \%)$ \\
Anemia + leukocytosis + & $57(11.4 \%)$ \\
thrombocytopenia & $40(8 \%)$ \\
Anemia & $23(4.6 \%)$ \\
Anemia + thrombocytopenia & $23(4.6 \%)$ \\
Albumin/globulin inversion + Ig increase & $17(3.4 \%)$ \\
Anemia + leukocytosis & $16(3.2 \%)$ \\
Leukocytosis & $11(2.2 \%)$ \\
Thrombocytopenia & $10(2 \%)$ \\
Anemia + leukopenia & $7(1.4 \%)$ \\
Polycythemia & $6(1.2 \%)$ \\
Thrombocytopenia + leukopenia & $4(0.8 \%)$ \\
Thrombocytosis + leukocytosis & \\
Clinical findings & $69(13.8 \%)$ \\
Acute leukemia remission evaluation & $41(8.2 \%)$ \\
Lymphoma staging & $37(7.4 \%)$ \\
Multiple myeloma remission evaluation & $17(3.4 \%)$ \\
Splenomegaly & $12(2.4 \%)$ \\
Chronic leukemia remission evaluation & $4(0.8 \%)$ \\
Fever & $23(4.6 \%)$ \\
Other & \\
\hline &
\end{tabular}

Ig: Immunoglobulin. 


\section{RESULTS}

Of the 500 patients included in the study, 274 $(54.8 \%)$ were male and $226(45.2 \%)$ were female, and the median age was $51.52 \pm 16.88(18-87)$ years. A bone marrow examination was performed for diagnosis in $382(76.4 \%)$ of the patients and for the evaluation of remission in 118 (23.6\%). The most common indication for a bone marrow examination was pancytopenia $(n=83,16.6 \%)$. The remaining indications are shown in Table-I. BMA was evaluated in 475 (95\%) of the patients included in the sample, while it could not be evaluated in the remaining $25(5 \%)$ patients due to insufficient particle sampling or poor staining. According to BMA evaluation, the most common diagnoses were hematological malignancies. Table-II presents the results of the BMA evaluations in all the patients. Among the 475 patients diagnosed based on the BMA evaluation, the diagnosis was consistent with the BMB report in $456(96 \%)$ cases (Table-III). For a total of $44(8.8 \%)$ cases, including $25(5 \%)$ that

Table-II: Results of bone marrow aspiration.

\begin{tabular}{lc}
\hline Diagnosis & $n(\%)$ \\
\hline Acute leukemia (AML + ALL) & $99(19.8 \%)$ \\
Remission evaluation: & $79(15.8 \%)$ \\
In remission & $30(6 \%)$ \\
Not in remission & $74(14.8 \%)$ \\
Normal bone marrow & $44(8.8 \%)$ \\
Multiple myeloma & $39(7.8 \%)$ \\
MDS & $25(5 \%)$ \\
Not evaluated & $22(4.4 \%)$ \\
CLL & $21(4.2 \%)$ \\
CML & $14(2.8 \%)$ \\
MPN other than CML & $10(2 \%)$ \\
Lymphoma infiltration & $9(1.8 \%)$ \\
Solid organ metastasis & $9(1.8 \%)$ \\
Hypocellular bone marrow & $6(1.2 \%)$ \\
Megaloblastic anemia & $6(1.2 \%)$ \\
Hairy cell leukemia & $6(1.2 \%)$ \\
ITP & $4(0.8 \%)$ \\
HLH & $3(0.6 \%)$ \\
Aplastic anemia & $A c u t e l y m-$ \\
\hline AML: Acute myeloid &
\end{tabular}

AML: Acute myeloid leukemia; ALL: Acute lymphocytic leukemia; MDS: Myelodysplastic syndrome; CLL: Chronic lymphocytic leukemia; CML: Chronic myeloid leukemia; MPN: Myeloproliferative neoplasia: ITP: Immune thrombocytopenic purpura; HLH: Hemophagocytic lymphohistiocytosis. could not be evaluated with BMA and 19 in which the $\mathrm{BMB}$ result was not consistent with the BMB report, the definitive diagnosis was made based on the BMB results, as shown in Table-IV.

\section{DISCUSSION}

Since acute and chronic leukemias show diffuse involvement in bone marrow, a BMA evaluation is usually sufficient when these malignancies are suspected. ${ }^{2}$ It is possible to differentiate Acute myeloid leukemia (AML) or Acute lymphocytic leukemia (ALL) according to the characteristics of blasts seen in the BMA. However, it may sometimes not be possible to distinguish acute leukemias by a morphological examination. Therefore, acute leukemias need to be subtyped for both treatment and follow-up purposes. In order to achieve this in the most accurate way, cells in blood samples taken by BMA should be evaluated with flow cytometry for immunophenotyping. ${ }^{4}$ In our study; flow

Table-III: Diagnostic agreement between BMA and BMB.

\begin{tabular}{lc}
\hline BMA diagnosis & $\begin{array}{c}\text { Agreement with } \\
\text { BMB diagnosis }\end{array}$ \\
\hline Acute leukemia (AML + ALL) & $99 / 99(100 \%)$ \\
Remission evaluation: & \\
In remission & $78 / 79(98.7 \%)$ \\
Not in remission & $30 / 30(100 \%)$ \\
Normal bone marrow & $69 / 74(93.2 \%)$ \\
Multiple myeloma & $44 / 44(100 \%)$ \\
MDS & $33 / 39(84.6 \%)$ \\
CLL & $22 / 22(100 \%)$ \\
CML & $21 / 21(100 \%)$ \\
MPN other than CML & $11 / 14(78.6 \%)$ \\
Lymphoma infiltration & $10 / 10(100 \%)$ \\
Solid organ metastasis & $9 / 9(100 \%)$ \\
Hypocellular bone marrow & $5 / 9(55.5 \%)$ \\
Megaloblastic anemia & $6 / 6(100 \%)$ \\
Hairy cell leukemia & $6 / 6(100 \%)$ \\
ITP (planned to undergo splenectomy) & $6 / 6(100 \%)$ \\
HLH & $4 / 4(100 \%)$ \\
Aplastic anemia & $3 / 3(100 \%)$ \\
Not evaluated & $0 / 25(0 \%)$ \\
\hline BMA Bone marrow aspiration BMB
\end{tabular}

BMA: Bone marrow aspiration; BMB: Bone marrow biopsy; AML: Acute myeloid leukemia, ALL: Acute lymphocytic leukemia; MDS: Myelodysplastic syndrome; CLL: Chronic lymphocytic leukemia; CML: Chronic myeloid leukemia; ITP: Immune thrombocytopenic purpura; MPN: Myeloproliferative neoplasia; HLH: Hemophagocytic lymphohistiocytosis. 
Table-IV: The results of patients not diagnosed with BMA but diagnosed with BMB.

\begin{tabular}{lc}
\hline Diagnosis & $44 / 500(\% 8.8)$ \\
\hline Normal bone marrow & $15(\% 3)$ \\
MDS & $9(\% 1.8)$ \\
Lymphoma infiltration & $6(\% 1.2)$ \\
Multiple myeloma & $3(\% 0.6)$ \\
Secondary metastasis & $3(\% 0.6)$ \\
Aplastic anemia & $2(\% 0.4)$ \\
MPN other than CML & $2(\% 0.4)$ \\
CMML & $2(\% 0.4)$ \\
LHH & $1(\% 0.2)$ \\
Megaloblastic anemia & $1(\% 0.2)$ \\
\hline
\end{tabular}

LHH: Hemophagocytic lymphohistiocytosis;

CMML: Chronic myelomonocytic leukemia.

cytometry was performed in all cases of acute and chronic leukemias. Flow cytometry is an effective method used in the diagnosis, differential diagnosis and follow-up of plasma cell neoplasms. In our study, we used flow cytometry in the differential diagnosis of multiple myeloma in patients with whom we considered plasma cell leukemia. In Eser A.'s study, the importance of flow cytometry, which is studied in the marrow blood sample taken with the first aspirate, is emphasized in the diagnosis and follow-up of multiple myeloma. ${ }^{5}$ It was easier to diagnose Chronic lymphocytic leukemia (CLL), Chronic myeloid leukemia (CML) and multiple myeloma with the evaluation of BMA due to the specific morphological appearance of bone marrow smear.

In a study by Bashawri, in which indications for a bone marrow examination and the most common diagnoses were determined in 1,813 patients presenting to a university hospital, the correlation between BMA and BMB diagnoses was evaluated. In that study, the most common indication for this examination was determined as the diagnosis and treatment of acute leukemia ( $\mathrm{n}=403,22.2 \%)$, and BMA was concluded to be an important diagnostic tool in many conditions, especially in hematological diseases. ${ }^{6}$ In our study, the most common diagnosis based on BMA was acute leukemia (AML + ALL) detected in $99(19.8 \%)$ patients, which was $100 \%$ consistent with the BMB report. In addition, in our study, there was 100\% agreement between BMA and $\mathrm{BMB}$ diagnoses in patients with chronic leukemia such as CLL and CML. The retrospective analysis of the data of Calvet et al. revealed that BMA contributed to the diagnosis and treatment of 40 $(20.7 \%)$ of 193 patients admitted to the intensive care unit. As a result, the authors concluded that BMA could significantly contribute to diagnosis and treatment in patients with or without any hematological malignancy during admission to the intensive care unit. ${ }^{7}$ In our study, the BMA evaluation directly contributed to the diagnosis and treatment of $200(40 \%)$ patients, including $186(37.2 \%)$ with malignant hematological diseases and 14 (2.8\%) with benign hematological diseases. In a study by Gilotara et al. in 100 cases, when the results of BMA and BMB assessment were compared, there was a $72.4 \%$ agreement. ${ }^{8}$ Similarly, Toi et al. compared the results of simultaneously performed BMA and BMA in 160 cases and reported that the results of the two procedures were consistent for $61.25 \% .{ }^{9}$ In the study of Puri et al., three hundred simultaneous BMA and BMB results were analyzed retrospectively. In this study, the overall agreement between BMA and BMB was $77.1 \%$. The reason for the high level of general agreement between BMA and BMB in our study was the high adequacy ratio of BMB materials. In this study, the highest overall concordance rates between BMA and $\mathrm{BMB}$ were CLL, CML and acute leukemias, which were similar to the rates in our study. ${ }^{10}$ In our study, the diagnostic correlation between BMA and $\mathrm{BMB}$ was high at $91.2 \%$ among the 500 patients included in the sample. In hematological malignant diseases such as acute leukemia, chronic leukemia, and multiple myelomas, the success of BMA was even higher, and the diagnostic agreement between BMA and BMB was $100 \%$.

In leukemias and other hematological malignancies with bone marrow involvement, in the interim period and after chemotherapy is completed, a re-examination of the bone marrow is required for the evaluation of remission. ${ }^{11}$ In our study, 79 patients were in remission according to BMA smear analysis. However, one of these patients was not in remission in BMB examination. In addition, 30 patients were found to be not in remission in both BMA and BMB examinations. In the study conducted by Puri et al., in the analysis of $\mathrm{BMA}$ and $\mathrm{BMB}$ results performed simultaneously in three hundred patients; In the evaluation of remission in patients with acute leukemia, CML and multiple myeloma, BMA and BMB were $100 \%$ compatible. Thus, in this study and in our study, BMA examination was found to be an effective diagnostic method in remission assessment. ${ }^{10}$

Although cell morphologies were adequately evaluated with BMA, in malignancies with focal 
involvement, such as lymphoma and multiple myeloma, the diagnosis is made based on BMB when there are not sufficient particles with aspiration or diagnostic cells. ${ }^{12,13}$ In our study, the results of BMA and BMB were fully consistent in the diagnosis of $44(8.8 \%)$ patients with multiple myeloma. However, three $(0.8 \%)$ patients with multiple myeloma could not be diagnosed with BMA due to focal involvement or insufficient particles, and the diagnosis was made using BMB. Similarly, for $10(2 \%)$ patients diagnosed with lymphoma, lymphoma infiltration was present in both BMA and $\mathrm{BMB}$, while three $(0.6 \%)$ patients had lymphoma involvement only in BMB due to focal involvement or insufficient bone marrow in BMA.

In a study conducted by Phillips et al., bone marrow involvement was found in 759 (62.5\%) patients in the bone marrow examination of a total of 1,215 patients diagnosed with lymphoma. ${ }^{14}$ In our study, the bone marrow examination was performed for staging purposes in 41 patients with a definitive diagnosis of lymphoma, and was observed in 10 of these patients in the BMA evaluation, while an additional six patients were detected to have bone marrow involvement in BMB. Therefore, in our study, $16(39 \%)$ of 41 patients who underwent lymphoma staging had bone marrow involvement and were considered stage- 4 . Metastasis of solid organ malignancies to the bone marrow is detected by the presence of non-hematological same type of cells in BMA examination..$^{15}$ In our study, solid organ metastasis was detected in the BMA evaluation of nine $(1.8 \%)$ cases. Similar to our study, Mansor et al. detected $2 \%$ solid organ metastases in the bone marrow examination of 1789 patients retrospectively. ${ }^{16}$

Limitations of the study: The design was retrospective and samples of a few cases could not be evaluated due to technical issues and problems about staining. However, our sample size is enough to determine the compatibilities between diagnosis via $\mathrm{BMA}$ and $\mathrm{BMB}$.

\section{CONCLUSION}

When the BMA evaluation is supported by flow cytometry and genetic analysis, almost all acute and chronic leukemias can be diagnosed. BMB is not necessary in the diagnosis of acute and chronic leukemia, except in cases where sufficient aspiration samples cannot be obtained for BMA evaluation. In the presence of the pre-diagnoses of lymphoma, multiple myeloma and solid organ metastasis, BMA should be performed together with BMB.
Conflict of interest: None.

\section{REFERENCES}

1. Licthman MA, Burns LJ. Clinical evaluation of the patient, 'Williams Hematology' (ninth edition), Kaushansky K \& (and 6 other Eds), New York, United States. 2016;pp 1-9.

2. Riley RS, Hogan TF, Pavot DR, Forysthe R, Massey D, Smith E, et al. A pathologist's perspective on bone marrow aspiration and biopsy: I. performing a bone marrow examination. J Clin Lab Anal. 2004;18(2):70-90. doi: 10.1002/jcla.20008

3. Lee SH, Erber WN, Porwit A, Tomonaga M, Peterson LC. International Council for Standardization In Hematology. ICSH guidelines for the standardization of bone marrow specimens and reports. Int J Lab Hematol. 2008;30(5):349-364. doi: 10.1111/j.1751553X.2008.01100.x

4. Anna Porwit, Clinical flow cytometry, 'Wintrobe's Clinical Hematology' (Fourteenth edition), Greer JP \& (and 8 other Eds), PA: Wolters Kluwer, Philadelphia, United States. 2019;pp 232-303.

5. Eser A. The importance of flow cytometry study with the first aspirate taken during bone marrow aspiration in the diagnosis of multiple myeloma and follow-up of minimal residual disease. Med Sci Discov. 2021;8(4):219-224.

6. Bashawri LA. Bone marrow examination Indications and diagnostic value. Saudi Med J. 2002;23(2):191-196.

7. Calvet L, Pereira B, Sapin A-F, Mareynat G, Lautrette A and Souweine $\mathrm{B}$. Contribution to diagnosis and treatment of bone marrow aspirate results in critically ill patients undergoing bone marrow aspiration: a retrospective study of 193 consecutive patients. J Intens Care. 2017;5(1):67. doi: 10.1186/s40560-017-0263-7

8. Gilotra M, Gupta M, Singh S, Sen R. Comparison of bone marrow aspiration cytology with bone marrow trephine biopsy histopathology: An observational study. J Lab Physicians. 2017;9(3):182-189. doi: 10.4103/JLP. JLP_117_16

9. Toi CP, Varghese RG'B, Rai R. Comparative Evaluation of Simultaneous Bone Marrow Aspiration and Bone Marrow Biopsy: An Institutional Experience. Indian J Hematol Blood Transfus. 2010;26(2):41-44. doi: 10.1007/s12288-010-0010-x

10. Puri V, Sharma P, Kotru M, Sikka M, Sharma S. Utility of simultaneous assessment of bone marrow aspirates and trephine biopsy sections in various haematological disorders. Iraqi J Hematol.2018;7(1):26-32. doi: 10.4103/ijh.ijh_33_17

11. Management of Haematological Malignancy, 'Hoffbrand's Essential Haematology' (Seventh edition), Hoffbrand AV \& Moss P AH (Eds), Wiley Blackwell, West Sussex, UK. 2016;pp:135-144.

12. Charles KS, Winfield DA, Angel C, Goepel J. Audit of bone marrow aspirates and trephine biopsies in multiple myeloma-a single centre study. Clin Lab Hematol. 2004;26(6):403-406. doi: 10.1111/j.13652257.2004.00646.x

13. Moid F, Depalma L. Comparison of relative value of bone marrow aspirates and bone marrow trephine biopsies in the diagnosis of solid tumour metastasis and Hodgkin Lymphoma. Arch Pathol Lab Med. 2005;129(4):497-501. doi: 10.1043/1543-2165(2005)129<497:CO RVOB $>2.0 . C O ; 2$.

14. Phillips L, Opie J. The utility of bone marrow sampling in the diagnosis and staging of lymphoma in South Africa. Int J Lab Hem. 2018;40(3): 276-283. doi: 10.1111/ijlh.12782.

15. Ozkalemkas F, Ali R, Ozkocaman V, Oscelik T, Ozanu OH. The bone marrow aspirate and biopsy in the diagnosis of unsuspected non hematologic malignancy. A clinical study of 19 cases. BMC Cancer. 2005; 1(5), 144. doi: 10.1186/1471-2407-5-144.16

16. Mansoor SN, Ali M, Mueez F, Nadeem SM, Nadeem W and Nadeem SM. Hematological Diagnosis in Children: Results of a 10 Year Long Cohort Based Upon Bone Marrow Examination. Pak J Med Health Sci. 2017;11(2): 664-667.

\section{Authors' Contributions:}

DA: Was responsible for the accuracy and integrity of the study.

DA, DS: Analyzed and interpreted the data, prepared the manuscript, performed the statistical analyses, and were responsible for the final editing. 